\title{
Cerebral Doppler and misrepresentation of flow changes
}

\author{
Marianne Thoresen, Kirsti Haaland, Petter Andreas Steen
}

\begin{abstract}
To determine whether cerebral blood flow velocity (CBFV) measurements were representative of cerebral blood flow (CBF) changes in pathological flow situations five newborn piglets were investigated. They underwent measurements of CBF by electromagnetic flowmetry on a modified common carotid artery where extracerebral branches were tied off simultaneously with Doppler recording either from the same precerebral or an intracerebral artery. The two methods agreed well within moderate carbon dioxide and blood pressure changes. During severe hypotension and hypertension Doppler overestimated CBF by 25-100\%. During transfusion of infected or incompatible blood the two methods differed in opposite directions with Doppler reading from $30-200 \%$ of $\mathrm{CBF}$. Transfusion of chilled blood caused CBFV to overestimate $15 \%$ and heated blood caused $20 \%$ underestimation. These results could be explained by diameter changes in response to variation in myogenic tone or vasoactive substances. CBFV measurements could be seriously misleading in severe clinical derangements where neonatal brain damage might occur. (Arch Dis Child 1994; 71: F103-F106)
\end{abstract}

Doppler ultrasound is increasingly used to evaluate the newborn cerebral circulation both in health and disease. We have developed an animal model for examining cerebral blood flow (CBF) using simultaneously electromagnetic flowmetry and Doppler ultrasound to measure cerebral blood flow velocity (CBFV) on a modified common carotid artery where extracerebral branches were tied off leaving the common carotid artery a true precerebral vessel. ${ }^{1}$ CBFV recordings were also made from an intracerebral artery through an artificial fontanelle. During normal physiological changes in flow, it is assumed that large arteries do not change their diameter. ${ }^{2}$ If the diameter as well as the angle of insonication are constant, then changes in CBFV reflect true changes in volume flow. ${ }^{3}$ With moderate carbon dioxide and mean arterial blood pressure (MABP) induced flow changes, the relative changes in CBF and CBFV are very similar and always in the same direction. ${ }^{4}$ As brain damage in the sick neonate is often due to ischaemia, ${ }^{5}$ measurement of cerebral haemodynamics in sick infants is important. However, the infants most at risk of cerebral damage are those subjected to processes which include hypotension, ${ }^{6}$ hypoxia, shock, septicaemia, and haemolytic disease. ${ }^{5}$ Vasoactive substances may be released in such conditions with unpredictable effects on cerebral arterial diameter. ${ }^{7}$ We used the two methods simultaneously to compare $\mathrm{CBFV}$ and $\mathrm{CBF}$ in overtly pathological situations where cerebral ischaemia might occur.

\section{Methods}

Five piglets with median age 1 day (range $0.5-4$ ) and median weight $2040 \mathrm{~g}$ (range 1500-2150) were anaesthetised with chloralose/urethane, paralysed, and artificially ventilated. ${ }^{8}$ Catheters were inserted in the umbilical artery and vein for MABP recording, blood sampling, and infusions. We intended to record CBF and CBFV from the internal carotid artery, however, access to this vessel requires extensive dissection which makes it impossible to place two transducers on to this vessel. We therefore turned the right common carotid artery into a precerebral vessel by ligating the external carotid artery and a small

Department of Paediatrics and Institute for

Experimental

Research, Ullevål

University Hospital,

Oslo, Norway

K Haaland

Institute for

Experimental

Research, Ullevål

University Hospital,

Oslo, Norway

P A Steen

Correspondence and reprint requests to:

Dr M Thoresen, Department

of Paediatric Research,

0027 Oslo, Norway.

Accepted 15 June 1994

Characteristics of the piglets

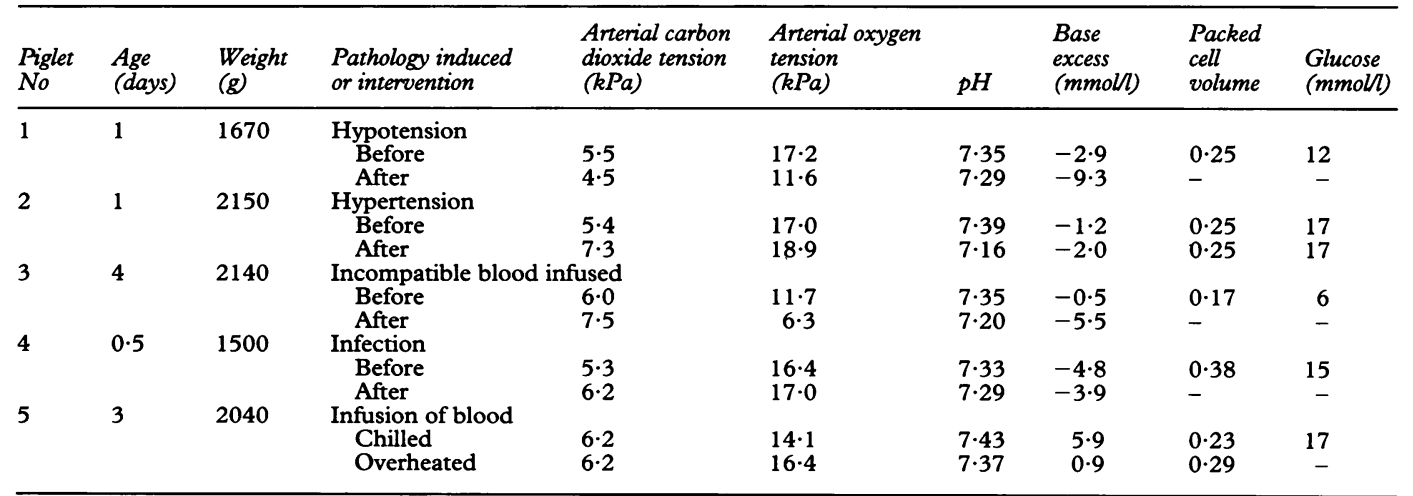



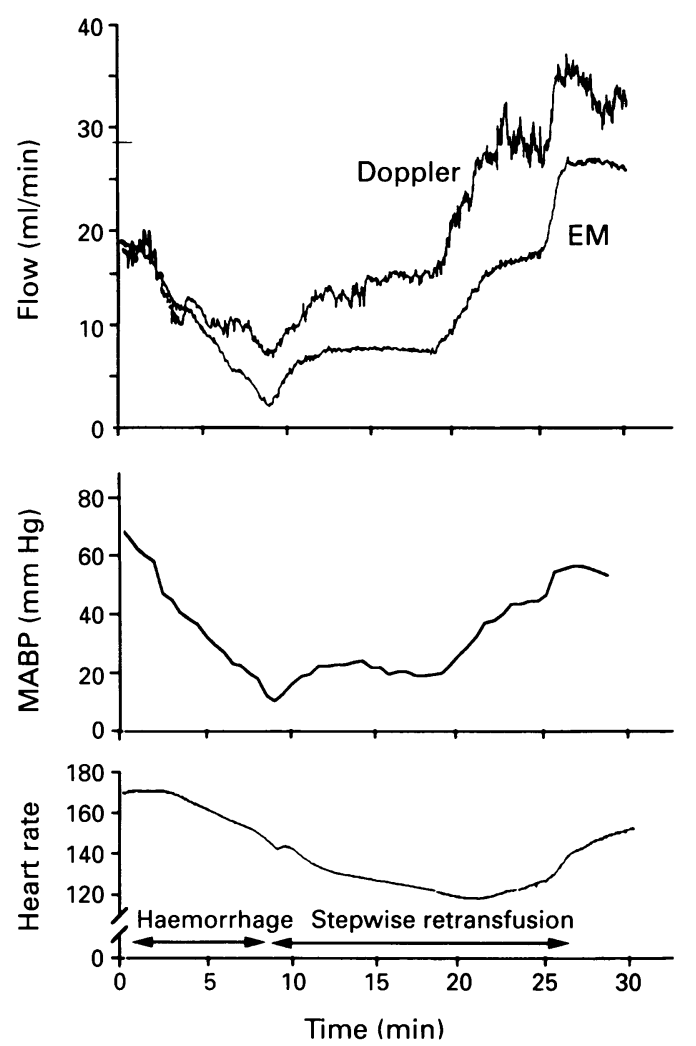

Figure 1 Upper panel show simultaneous and continuous recording of estimated Doppler flow and $C B F$ by

electromagnetic flowmetry (EM) on the modified common carotid artery during haemorrhage and retransfusion in piglet 1. Doppler velocities are calculated into flow by using an estimated diameter of $1.5 \mathrm{~mm}$ and a measured angle of insonication of $30^{\circ}$. The corresponding $M A B P$ and heart rate is displayed in the middle and lower panel respectively.

branch on the internal carotid artery (the occipital artery). That this modified common carotid supplied the brain was validated by dye injection. An electromagnetic flowmeter probe was fitted around the right common carotid artery (type 372 Nycotron A/S Norway). The flowmeter was calibrated in situ at the end of the experiment infusing a known volume of blood over a known period of time. Precerebral $\mathrm{CBFV}$ was recorded from the modified common carotid artery with the Doppler transducer $(10 \mathrm{MHz}$ pulsed, Vingmed SD 100 , Vingmed Sound A/S Norway) positioned in a stereotactic holder and insonicating the artery at an angle of $30^{\circ}$. Intracerebral CBFV (cortical artery at $1 \mathrm{~cm}$ depth with anatomical localisation corresponding to the middle cerebral artery) were recorded through an artificial fontanelle made above the right side of the brain. Individual characteristics of the five piglets are given in the table.

CBFV recordings were made from the precerebral artery in piglet $1,2,3$, and 5 . In piglet 4 the CBFV recording was obtained from an intracerebral artery. Pathological flow changes were induced for piglet 1 by severe hypotension with $\mathrm{rapid}(3.3 \mathrm{ml} / \mathrm{kg} / \mathrm{min})$ withdrawal of $50 \mathrm{ml}$ of blood. In piglet 2 , hypertension was induced by rapid $(2.7 \mathrm{ml} / \mathrm{kg} / \mathrm{min})$ transfusion of $95 \mathrm{ml}$ of cross matched pig blood. In piglet 3, $8 \mathrm{ml}$ of incompatible (human) blood were infused. In piglet $4,18 \mathrm{ml}$ of infected (and thus haemolysed) blood were infused. In piglet $5,6 \mathrm{ml}$ of blood that had been chilled to $10^{\circ} \mathrm{C}$ were given. When $\mathrm{CBF}$ and $\mathrm{CBFV}$ had normalised, $6 \mathrm{ml}$ of blood that had been overheated to $45^{\circ} \mathrm{C}$ were given. At the end of the experiment, the animals were killed by intravenous potassium chloride.

\section{Results}

During haemorrhage and stepwise retransfusion the relative changes in Doppler CBFV recorded from a modified common carotid artery closely followed the relative changes in $\mathrm{CBF}$ until the MABP was reduced by $50 \%$ (piglet 1, fig 1). Thereafter the Doppler overestimated the flow values by $100 \%$ at the most during severe hypotension. The overestimation was reduced to $25 \%$ by the end of retransfusion. The heart rate (beats/min) decreased during hypotension as shown in the lower panel.

In fig 2 transfusion of $95 \mathrm{ml}$ blood over 17 minutes at a constant rate gradually
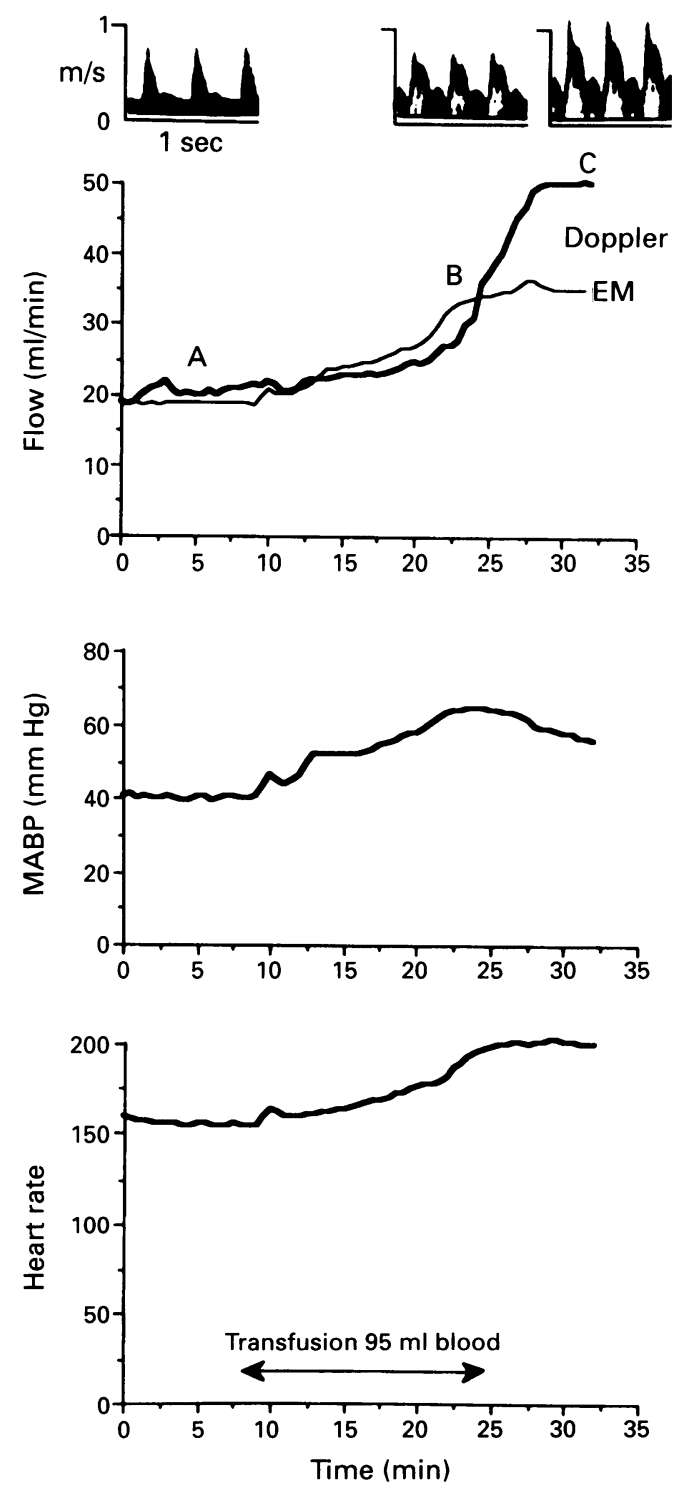

Figure 2 Simultaneous and continuous recording of estimated Doppler flow and CBF by electromagnetic flowmetry (EM) on the precerebral carotid artery during transfusion of $95 \mathrm{ml}$ cross matched pig blood. Doppler spectra from three flows situations as indicated $(A, B, C)$ are inserted at the top. The corresponding MABP and heart rate are displayed in the middle and lower panel respectively. 

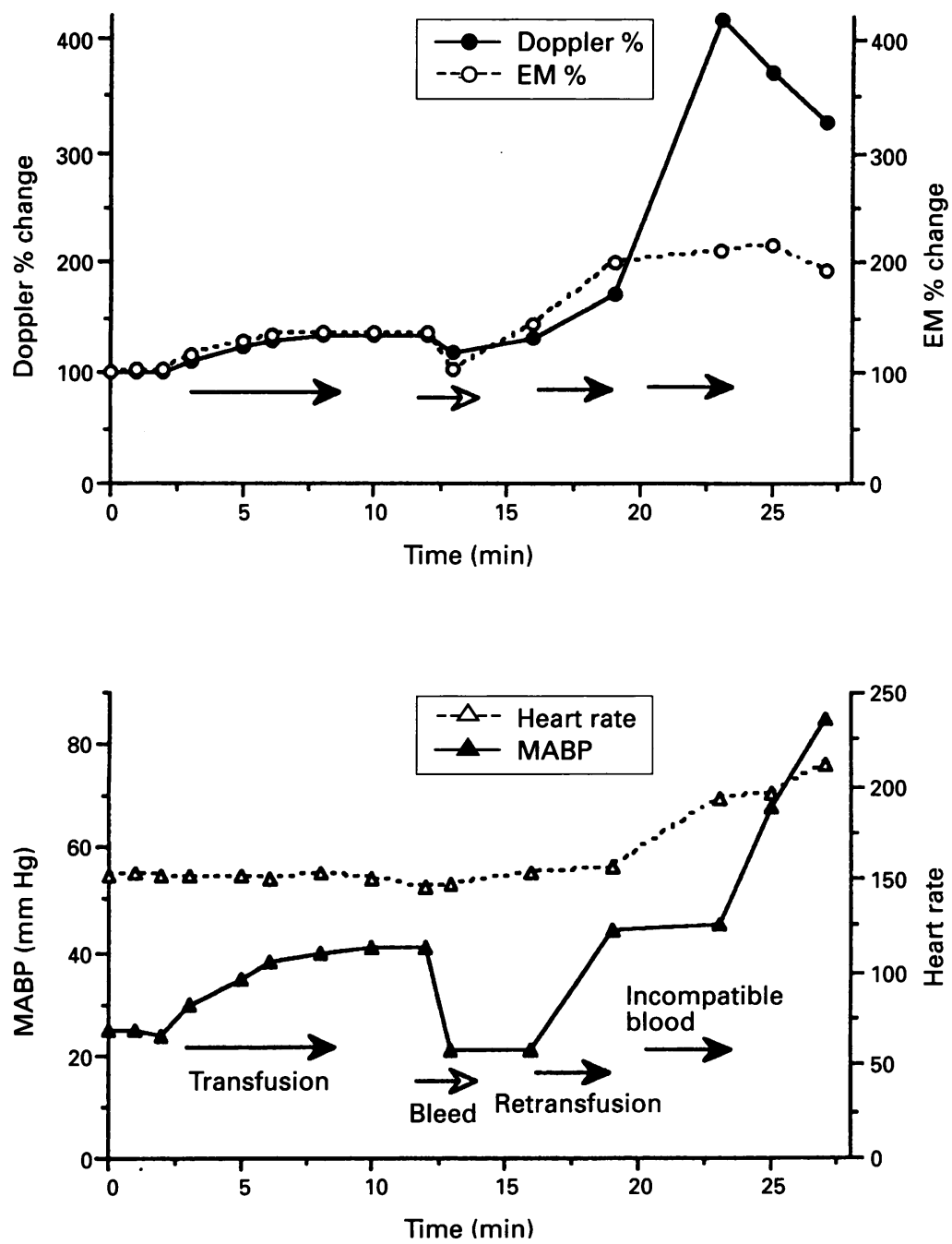

Figure 3 Doppler $C B F V$ from an intracerebral artery and $C B F$ by electromagnetic flowmetry (EM) from the modified common carotid artery were recorded simultaneously during transfusion, haemorrhage, and retransfusion of cross matched pig blood followed by transfusion of $8 \mathrm{ml}$ of human blood (piglet 3). The MABP and heart rate are shown in the lower panel. to maximum response by Doppler CBFV (piglet 5, fig 5). During infusion of overheated blood there was an immediate and transient $25 \%$ increase in both heart rate, $M A B P, C B F$, and $C B F V$. While heart rate, MABP, and CBF thereafter normalised, CBFV continued to fall thus underestimating CBF by $15 \%$ at the end of the recording.

\section{Discussion}

Although it is suggested that Doppler flow velocity measurements may underestimate changes in CBF as directly measured, ${ }^{9}$ discrepancies of such magnitude as shown here and in opposite directions to directly measured CBF have not been previously reported. The uncertainty in the CBF estimate from the CBFV measurements (cross sectional vessel area $\times$ average mean velocity) is probably caused by changes in vessel diameter, as a constant vessel diameter is a prerequisite for the estimation. As it is not known which pathological situations might change the vessel diameter in the human infant we chose physiological as well as extremely pathological stimuli for this comparative study between CBF and CBFV.

Within the normal physiological range of carbon dioxide tension and MABP we have recently reported good correlation between changes in directly measured CBF and CBFV in the same model. ${ }^{14}$ The present results indicate that this may not hold true in grossly pathophysiological situations.

When $\mathrm{CBF}$ changes were induced by changes in MABP in response to large

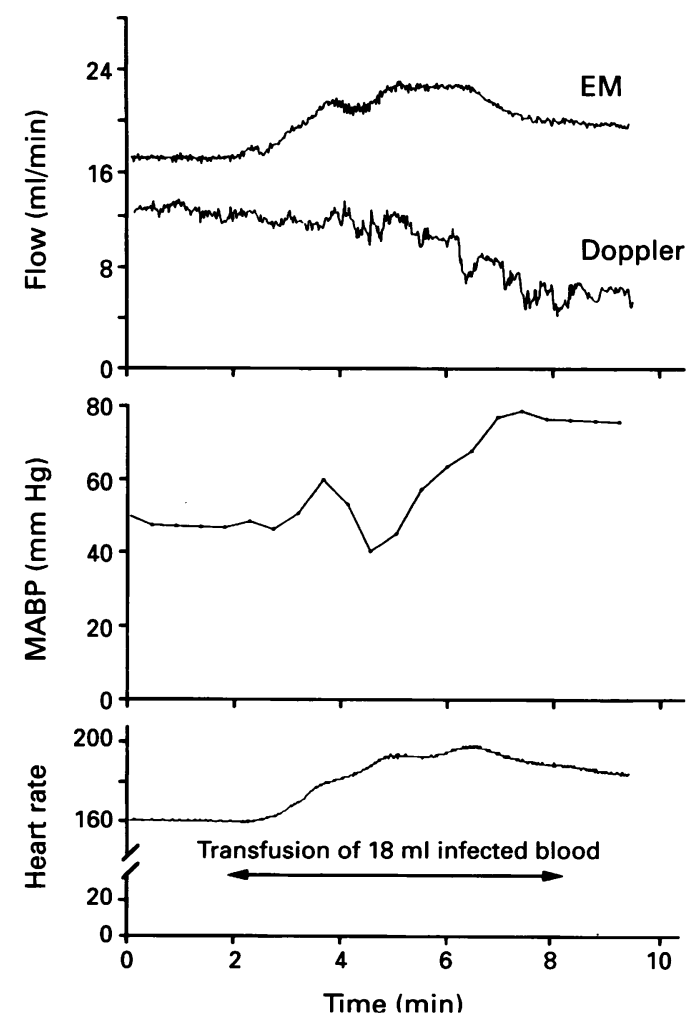

Figure 4 Simultaneous Doppler $C B F V$ and $C B F$ by electromagnetic flowmetry (EM) measurements on the carotid artery in piglet $4 ; 18 \mathrm{ml}$ of haemolysed and infected blood were transfused during six minutes. $M A B P$ and heart rate are shown in the lower panel. 

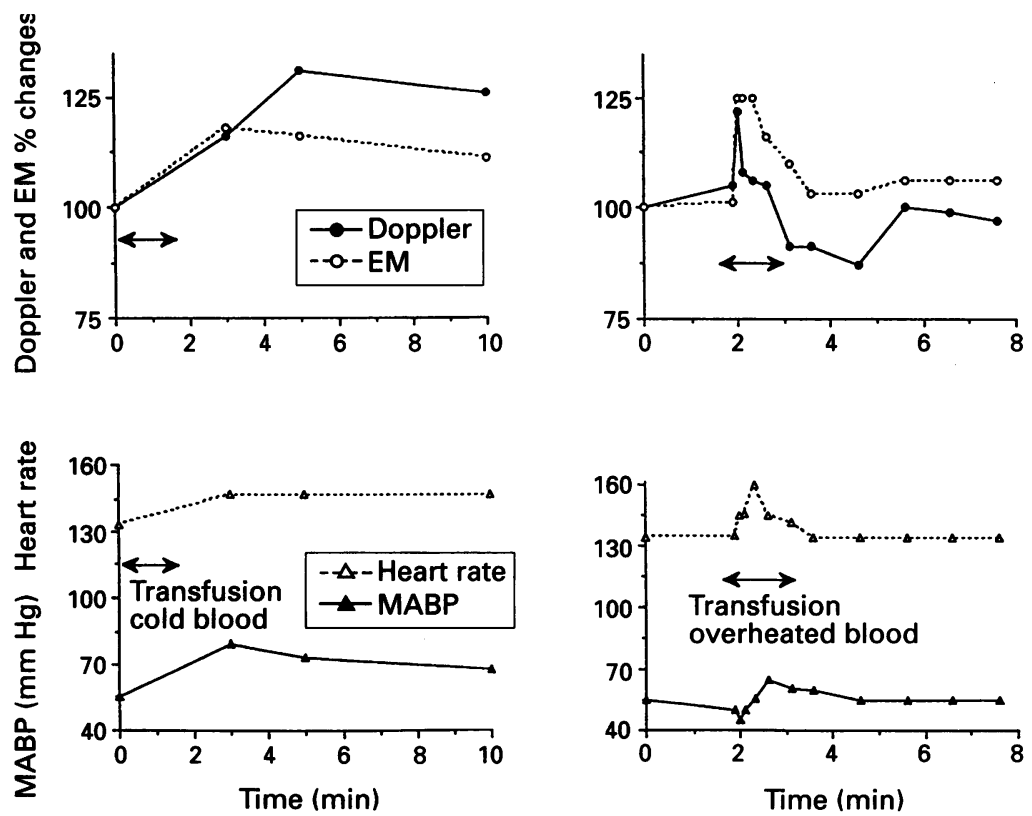

Figure 5 Upper left graph show the time course of $C B F V$ and $C B F$ by electromagnetic flowmetry (EM) recording next to each other on the same precerebral modified common carotid artery during transfusion of $6 \mathrm{ml}$ of cold autologous blood (piglet 5). The starting values before transfusion at time 0 minutes were normalised to $100 \%$. In the lower left graph is shown the corresponding heart rate and $M A B P$ changes. The two graphs to the right show corresponding $C B F$ and $C B F V$ recordings in response to transfusion of overheated blood (piglet 5). In the lower panel the corresponding heart rate and $M A B P$ changes are shown.

changes in blood volume in this neonatal piglet model which has inadequate autoregulatory capacity, ${ }^{4}$ the Doppler consistently overestimated CBF during hypotension and also after a large transfusion. Variation in myogenic stretch induced tone is considered an important element of cerebral autoregulation. ${ }^{10}$ Increased pressure leads to stretch and subsequent vessel contraction. This can explain the discrepancy between the CBFV and CBF changes during increases in MABP with a decrease in vessel diameter due to vessel contraction. The rapidly induced hypotension also resulted in CBFV overestimating CBF. When the MABP fell to as little as $10 \mathrm{~mm} \mathrm{Hg}$ there was no pressure to dilate the.vessel and thus the CBFV overestimated $\mathrm{CBF}$.

Moderate discrepancies between CBFV and CBF occurred with infusion of cold and heated blood which could be explained by vessel vasoconstriction due to cold blood and vasodilatation due to hot blood. It is important to note in all figures that the time courses of CBF and CBFV changes are usually not simultaneous. There is growing evidence that there is prolonged (several minutes) and cyclical diameter changes in response to different stimuli. ${ }^{11} 12$

With infusion of contaminated blood CBFV and CBF were observed to change in opposite directions - an increase in electromagnetic flowmeter and decrease in Doppler while there was an increase in blood pressure. Absence of autoregulation with a pressure passive circulation and a decreased vessel diameter could explain this result. A combination of reduced CBFV and increased MABP has been found in newborns treated with indomethacin. ${ }^{13}$

Although infusion of contaminated blood is clinically a rare condition, ${ }^{14}$ we used this as a way of infecting the animal at a definite point in time to allow us to follow the changes. One can only speculate to what degree septicaemia evolving over a longer period of time would release vasoactive substances ${ }^{7}$ and affect vessel diameter.

Although one aims to transfuse blood at body temperature, in emergency situations refrigerated blood may sometimes be substantially below $37^{\circ} \mathrm{C}$ while transfused. Attempts to warm blood rapidly can result in blood being overheated. Thus our pathological situations are not impossible in the clinical situation.

In one animal where the CBFV was recorded from an intracerebral artery, a small volume of incompatible blood was infused and a rapid $200 \%$ increase in CBFV compared with $\mathrm{CBF}$ was seen. During MABP changes between 30 and $55 \mathrm{~mm} \mathrm{Hg}$ intracerebral CBFV followed precerebral CBF closely. Although most of the procedures performed in this study are not physiological, our intention was to show that it is possible to induce substantial diameter changes in large precerebral and smaller intracerebral arteries.

In severe pathophysiological derangements, where cerebral ischaemia is likely, investigators using cerebral Doppler should be aware of this potential source of error in the estimation of the magnitude and direction of cerebral blood flow changes.

$\mathrm{KH}$ was supported by the Norwegian Research Council. MT is grateful for grants from the Norwegian Research Council, the Laerdal Foundation for Acute Medicine and The Odd Fellow Foundation.

1 Haaland K, Karlsson B, Skovlund E, Thoresen M. Simultaneous measurements of cerebral circulation with electromagnetic flowmetry and Doppler velocity in the newborn pig. Pediatr Res (in press)

2 Kontos HA, Wei EP, Navari RM, Levasseur JE, Rosenblum WI, Patterson JLJ. Response of cerebral arteries and arterioles to acute hypotension and hypertension. $A m^{f}$ Physiol 1978; 234: H371-83.

3 Guldvog I, Kjærnes M, Thoresen M, Walløe L. Blood flow in arteries determined transcutaneously by an ultrasonic in arteries determined transcutaneously by an ultrasonic measurements on the exposed vessels. Acta Physiol Scand 1980; 109: 211-6.

4 Haaland K, Karlsson B, Skovlund E, Lagercrantz H, Thoresen M. Postnatal development of the cerebral blood flow velocity response to changes in $\mathrm{CO} 2$ and $\mathrm{MABP}$ in the piglet. F Dev Physiol (in press).

5 De Vries LM, Larroche JC, Levene MI. Cerebral ischaemic lesions. In: Levene MI, Bennet MJ, Punt J, eds. Fetal and neonatal neurology and neurosurgery. Edinburgh: Churchill Livingstone, 1988: 346-55.

6 Miall-Allen VM, De Vries LS, Whitelaw AGL. Mean arterial blood pressure and neonatal cerebral lesions. Arch Dis Child 1987; 62: 1068-9.

7 Heyderman RS, Klein GS, Shennan GI, Levin M Deficiency of prostacyclin production in meningococcal Deficiency of prostacyclin production in
shock. Arch Dis Child 1991; 66: 1296-9.

8 Thoresen M, Whitelaw A. The effects of acetazolamide on $\mathrm{CBF}$ velocity and $\mathrm{CO}_{2}$ elimination in normotensive and hypotensive newborn piglets. Biol Neonate 1990; 58: 200-7.

9 Lundell BPW, Kennedy KA, Lindstrom DP, Sundell H, Stahlman MT. Intracranial Doppler flow velocimetry compared with extracranial carotid blood flow measurements. Acta Paediatrica Scandinavica 1986; 329 (suppl): 127-33.

10 Wei EP, Kontos HA. Response of cerebral arterioles to increased venous pressure. Am $\mathcal{f}$ Physiol 1982; 243: H442-7.

11 Bevan JA. Resistance artery tone is influenced independently by pressure and by flow. Blood Vessels 1990; 27: 202-7.

12 Garcia-Roldan JL, Bevan JA. Flow-induced constriction and dilation of cerebral resistance arteries. Circ Res 1990; 66: $1445-8$.

13 Cowan F. Indomethacin, patent ductus arteriousus, and cerebral blood flow. F Pediatr 1986; 109: 341-4.

14 Foreman NK, Wang WC, Cullen EJ, Stidham GL, Pearson MS, Shenep JL. Endotoxic shock after transfusion of conMS, Shenep JL. Endotoxic shock after transfusion of conPediatr Infect Dis f 1991; 10: 624-5. 